\title{
Cisplatin or carboplatin? Neutrophil to lymphocyte ratio may serve as a useful factor in small cell lung cancer therapy selection
}

\author{
ZHANGCHI PAN $^{1^{*}}$, LU ZHANG $^{2^{*}}$, CHEN LIU $^{3}$, XIAOBING HUANG $^{1}$, \\ SONGFEI SHEN ${ }^{1}$, XIAOYAN LIN ${ }^{1}$ and CHUNMEI SHI ${ }^{1,4,5}$
}

\begin{abstract}
Departments of ${ }^{1}$ Oncology and ${ }^{2}$ Clinical Laboratories, Fujian Medical University Union Hospital; ${ }^{3}$ Department of Oncology, Fujian Magaw Memorial Hospital; ${ }^{4}$ Molecular Biology Laboratory, Fujian Medical University Stem Cell Research Institute; ${ }^{5}$ Fujian Key Laboratory of Translational Cancer Medicine, Fujian Medical University, Fuzhou, Fujian 350001, P.R. China
\end{abstract}

Received June 4, 2018; Accepted April 15, 2019

DOI: $10.3892 / 01.2019 .10459$

\begin{abstract}
The present study aimed to investigate the significance of the neutrophil to lymphocyte ratio (NLR) in peripheral blood of patients with small cell lung cancer (SCLC) when selecting a first-line treatment. A total of 73 patients with SCLC who had complete clinical data and sought treatment at Fujian Medical University Union Hospital between January 2014 and May 2016 were included. Data were retrospectively analyzed, utilizing a receiver operating characteristic curve to determine the NLR cut-off value. Out of the 73 patients, 39 were classified as high-NLR (NLR $\geq 3.80$ ) and 34 as low-NLR (NLR <3.80). Compared with the high-NLR group, patients in the low-NLR group had a longer progression free survival (PFS); however, there was no statistically significant difference in overall survival (OS) time. Patients with a high NLR had a significantly longer PFS $(\mathrm{P}=0.021)$ and $\mathrm{OS}$ time $(\mathrm{P}=0.042)$ when treated with a etoposide/cisplatin (EP) therapy regimen, compared with those treated with etoposide/carboplatin (EC). PFS was the longest in the high-NLR patients with limited stage (LS; $\mathrm{P}=0.002$ ). Among the patients receiving the EC regimen, the PFS of the low-NLR group was significantly longer compared with the high-NLR group $(\mathrm{P}=0.003)$. Patients in the low-NLR group who received thoracic radiotherapy had a longer PFS $(\mathrm{P}=0.011)$, when comparing patients in the low-NLR group who did not receive thoracic radiotherapy, and within this group the therapeutic effect of radiation was the greatest in LS patients. Compared with the high-NLR group, the low-NLR group patients who received cranial radiotherapy
\end{abstract}

Correspondence to: Dr Chunmei Shi, Department of Oncology, Fujian Medical University Union Hospital, Building 2, 29 Xinquan Road, Fuzhou, Fujian 350001, P.R. China

E-mail: scmfz@qq.com

${ }^{*}$ Contributed equally

Key words: small cell lung cancer, neutrophil to lymphocyte ratio, chemotherapy, radiotherapy, progression free survival, overall survival had a significantly longer PFS $(\mathrm{P}=0.039)$. For the initial evaluation of patients with SCLC, pre-treatment NLR may be of significance for selecting first-line chemotherapy agents. As the present study was retrospective and investigated a limited number of patients, further research and prospective studies are warranted.

\section{Introduction}

Lung cancer is a common type of malignancy with significant mortality (1). Some researchers hold the opinion that small cell lung cancer (SCLC) is systemic from initial development (2). Due to its fast progression, poor prognosis and the tendency towards whole body metastasis at an early stage, the majority of patients are diagnosed at an advanced state of the disease (2). Only 30-40\% of patients are in the limited stage (LS), which means in the ipsilateral hemithorax and within a single radiotherapy region, prior to treatment (3). By combining surgery, radiotherapy and chemotherapy, LS patients may achieve a 15-20 month median survival time, and $20-40 \%$ have a 2 -year survival time (3). Certain extensive stage (ES) patients may only receive supportive treatment due to extensive metastasis and a poor performance status, thus resulting in an even shorter survival time (4). The median survival time for patients with SCLC is 2-4 months if the disease is left untreated, and the 2-year survival rate is $~ 5 \%$ (3). Over the past 15 years, the median and 5-year survival rate of patients with SCLC has not significantly improved (5).

At present, etoposide/carboplatin (EC) and etoposide/cisplatin (EP) therapy regimens are the two first line regimens for the treatment of SCLC (6). Although similar in efficacy, the two regimens have different toxicity profiles. Cisplatin is associated with adverse gastrointestinal effects, neurotoxicity and renal function impairment, and its administration requires a hydration regimen. Carboplatin is associated with myelosuppression (7-9).

The occurrence and progression of tumors is often accompanied by an intratumoral inflammatory response, which suppresses anti-tumor immunity (10). It has been demonstrated that this inflammatory response may serve an important role in the development and progression of lung cancer (10). The 
neutrophil to lymphocyte ratio (NLR) in peripheral blood serves as an inflammatory marker, representing the absolute value of the ratio of neutrophil count to lymphocyte count. As a routine hematological test, it is convenient. Additionally, it is associated with patient prognosis in different types of tumors, such as bladder cancer, hepatocellular carcinoma and non-small cell lung cancer (11-16). However, the prognostic value of NLR in SCLC requires further research. Currently, indicators that aid in the selection of first line treatment for patients with SCLC are limited. The present study retrospectively analyzed the relationship between the NLR and the progression-free survival (PFS) in 73 cases of SCLC in order to investigate the significance of the NLR when selecting a first-line treatment.

\section{Materials and methods}

Clinical data. A total of 73 SCLC cases with relatively complete clinical data were reviewed using the electronic medical records and registration database at the Fujian Medical University Union Hospital (Fuzhou, China). The patients had sought treatment at the aforementioned hospital between January 2014 and May 2016. The present study was approved by the Institutional Review Board (IRB) of Fujian Medical University Union Hospital (IRB no. 2017KY084) and informed consent was obtained from the patients. All patients were diagnosed with SCLC by pathological examination; lung tumor histopathological examination was used to confirm small cell carcinoma and immunohistochemical examination confirmed compliance with small cell lung cancer. The exclusion criteria were as follows: i) Second primary tumor; ii) diseases that may result in hematological changes (including lymphoma, leukemia and bone marrow dysplasia syndrome); iii) chronic diseases (including cardiovascular disease, diabetes, asthma and chronic obstructive pulmonary disease); iv) histological mixed-type tumors with adenocarcinoma and other histological types (Such as lung squamous cell carcinoma, alveolar carcinoma, mesenchymal sarcoma, and large cell carcinoma); and v) no exposure to prophylactic cranial irradiation. Among the 73 patients, 69 were male (94.50\%, mean age 61.43 years, age range $39.00-83.00$ years) and 4 were female $(5.50 \%$, mean age 65.25 years, age range 53.00-75.00 years). Data were collected from routine blood tests, including neutrophil and lymphocyte counts and levels of serum albumin and lactic dehydrogenase, performed during the initial diagnosis. Additionally, the first-line therapeutic regimen used was recorded for each patient. The follow-up time was set from the initial diagnosis to August 31st 2017. Information regarding PFS and overall survival (OS) time was acquired via inpatient and outpatient medical records and telephone follow-up. PFS was calculated from the date of first diagnosis to the onset of disease progression [According to RECIST 1.1 solid tumor efficacy evaluation criteria (17)] or the last follow-up. The OS time was defined as the period from diagnosis to mortality (OS time study endpoint) or the last follow-up.

Research methods. The PFS data were divided into two groups: Low PFS $(<4.50)$ and high PFS $(\geq 4.50)$ according to the PFS median of 4.50. An NLR cut-off value of 3.80 was
Table I. Clinical and hematological characteristics of patients with small cell lung cancer.

\begin{tabular}{lrc}
\hline Variable & Mean & Range \\
\hline Age (years) & 61.64 & $39.00-83.00$ \\
Sex (male/female) & 61.64 & $39.00-83.00$ \\
PFS (months) & 5.02 & $1.00-16.00$ \\
OS time (months) & 13.15 & $1.00-63.00$ \\
BMI $\left(\mathrm{kg} / \mathrm{m}^{2}\right)$ & 21.65 & $17.72-25.61$ \\
Lymphocytes count $\left(10^{9} / \mathrm{l}\right)$ & 2.01 & $0.46-26.80$ \\
Neutrophil count $\left(10^{9} / \mathrm{l}\right)$ & 5.41 & $1.27-11.90$ \\
Hemoglobin $(\mathrm{g} / \mathrm{l})$ & 131.75 & $93.00-169.00$ \\
RBC count $\left(10^{12} / \mathrm{l}\right)$ & 4.31 & $3.14-5.87$ \\
NLR & 3.81 & $0.27-13.63$ \\
\hline
\end{tabular}

PFS, progression-free survival; OS, overall survival; BMI, body mass index; RBC, red blood cell; NLR, neutrophil to lymphocyte ratio.

selected during initial diagnosis by means of a receiver operating characteristic (ROC) curve used to calculate Youden's index. The patients were subsequently divided into two groups: Low-NLR (NLR <3.80) and high-NLR $($ NLR $\geq 3.80)$. Based on the staging method of the Veterans Administration Lung Study Group of the United States (18), patients with SCLC were further classified into two groups: LS and ES. Statistical methods were used to analyze the associations between NLR, the choice of first-line therapeutic regimen, PFS and OS time.

Statistical analysis. Clinical data are presented as the mean \pm standard deviation or median (range). All analyses were performed using SPSS software (version 19; IBM Corp., Armonk, NY, USA). ROC curve analysis was used to determine the cut-off value for the NLR. The Chi-square test was used to compare the different clinical characteristics in the groups, and a t-test was used to compare the NLR between groups. The Kaplan-Meier method was used to compare survival time differences between the high- and low-NLR groups, and survival differences between patient groups were analyzed using the log-rank test. Stratified analysis was performed between LS and ES patients in order to compare the prognoses associated with different therapeutic regimens. $\mathrm{P}<0.05$ was considered to indicate a statistically significant difference. All P-values were two-tailed.

\section{Results}

Association between pre-treatment NLR and clinical characteristics. The 73 SCLC cases were divided into 39 high-NLR cases and 34 low-NLR cases. The clinical and hematological characteristics of the patients are presented in Table I. The differences in NLR between groups, following stratification by several clinical characteristics including age, sex and cancer stage, were not statistically significant $(\mathrm{P}>0.05$; Table II, Among the 73 patients, we could not collect smoking history data, stage, ECOG performance status, brain metastases, lung metastases, bone metastases, pleural metastasis, adrenal 
Table II. Association between the NLR and the clinicopathological characteristics of patients with SCLC.

\begin{tabular}{|c|c|c|c|c|c|c|c|c|}
\hline Characteristic & NLR & $\mathrm{t}$ & P-value & $\mathrm{n}$ & High NRL & Low NRL & $\chi^{2}$ & P-value \\
\hline Age at diagnosis (years) & & 0.336 & 0.738 & & & & 0.053 & 0.817 \\
\hline$<60$ & $3.94 \pm 3.12$ & & & 30 & 16 & 14 & & \\
\hline$\geq 60$ & $3.72 \pm 2.31$ & & & 43 & 23 & 18 & & \\
\hline Sex & & 0.93 & 0.761 & & & & 0.42 & 0.838 \\
\hline Male & $3.83 \pm 2.68$ & & & 69 & 37 & 30 & & \\
\hline Female & $3.59 \pm 2.78$ & & & 4 & 2 & 2 & & \\
\hline Smoking & & -0.218 & 0.828 & & & & 0.525 & 0.469 \\
\hline No & $3.54 \pm 2.26$ & & & 22 & 14 & 8 & & \\
\hline Yes & $3.69 \pm 2.64$ & & & 44 & 25 & 21 & & \\
\hline Stage & & 0.076 & 0.940 & & & & 0.053 & 0.817 \\
\hline LS-SCLC & $3.85 \pm 2.90$ & & & 29 & 16 & 14 & & \\
\hline ES-SCLC & $3.79 \pm 2.52$ & & & 37 & 23 & 18 & & \\
\hline ECOG performance status & & 1.127 & 0.288 & & & & 1.127 & 0.288 \\
\hline 1 Point & $3.04 \pm 1.44$ & & & 34 & 22 & 14 & & \\
\hline 2 Point & $4.61 \pm 3.35$ & & & 32 & 17 & 18 & & \\
\hline Brain metastases & & 0.062 & 0.804 & & & & 1.37 & 0.243 \\
\hline No & $3.54 \pm 2.35$ & & & 62 & 35 & 31 & & \\
\hline Yes & $3.84 \pm 2.70$ & & & 4 & 4 & 1 & & \\
\hline Lung metastases & & 0.233 & 0.816 & & & & 0.059 & 0.808 \\
\hline No & $3.86 \pm 2.89$ & & & 46 & 27 & 23 & & \\
\hline Yes & $3.70 \pm 2.09$ & & & 20 & 12 & 9 & & \\
\hline Bone metastases & & 0.092 & 0.927 & & & & 0.004 & 0.951 \\
\hline No & $3.83 \pm 2.65$ & & & 51 & 29 & 24 & & \\
\hline Yes & $3.77 \pm 2.78$ & & & 15 & 10 & 8 & & \\
\hline Pleural metastasis & & 1.670 & 0.397 & & & & 0.59 & 0.442 \\
\hline No & $3.70 \pm 2.60$ & & & 63 & 38 & 30 & & \\
\hline Yes & $6.30 \pm 3.64$ & & & 3 & 1 & 2 & & \\
\hline Adrenal metastasis & & 0.080 & 0.936 & & & & 0.42 & 0.838 \\
\hline No & $3.82 \pm 2.71$ & & & 63 & 37 & 30 & & \\
\hline Yes & $3.71 \pm 1.96$ & & & 3 & 2 & 2 & & \\
\hline LDH, IU/1 & & -1.602 & 0.114 & & & & 1.904 & 0.168 \\
\hline$\leq 245$ & $3.11 \pm 2.08$ & & & 26 & 19 & 10 & & \\
\hline$>245$ & $4.05 \pm 2.57$ & & & 38 & 19 & 20 & & \\
\hline Albumin, g/l & & 1.127 & 0.288 & & & & 0.342 & 0.559 \\
\hline$<37.5$ & $4.16 \pm 3.15$ & & & 31 & 18 & 17 & & \\
\hline$\geq 37.5$ & $3.48 \pm 2.08$ & & & 35 & 21 & 15 & & \\
\hline $\mathrm{NSE}, \mathrm{ng} / \mathrm{ml}$ & & -0.416 & 0.679 & & & & 0.223 & 0.637 \\
\hline$<16.3$ & $3.53 \pm 1.87$ & & & 13 & 6 & 7 & & \\
\hline$\geq 16.3$ & $3.86 \pm 2.65$ & & & 40 & 22 & 19 & & \\
\hline $\mathrm{CEA}, \mathrm{ng} / \mathrm{ml}$ & & 1.130 & 0.263 & & & & 2.302 & 0.129 \\
\hline$<5.0$ & $3.95 \pm 2.47$ & & & 37 & 19 & 20 & & \\
\hline$\geq 5.0$ & $3.23 \pm 2.42$ & & & 25 & 17 & 8 & & \\
\hline $\mathrm{MKI}^{\mathrm{a}}, \%$ & & -0.466 & 0.642 & & & & 0.34 & 0.523 \\
\hline$\leq 90$ & $3.70 \pm 2.46$ & & & 45 & 24 & 19 & & \\
\hline$>90$ & $4.00 \pm 2.99$ & & & 21 & 15 & 13 & & \\
\hline
\end{tabular}

${ }^{a}$ Was contained within the patient's pathological profile. $t, t$ test value; $n$, number; NLR, neutrophil to lymphocyte ratio; SCLC, small-cell lung cancer; LS-SCLC, limited stage small-cell lung cancer; ES-SCLC, extensive stage small-cell lung cancer; ECOG, Eastern Cooperative Oncology Group; LDH, lactate dehydrogenase; NSE, neuron-specific enolase; CEA, carcinoembryonic antigen; MKI67, marker of proliferation Ki-67. Among the 73 patients, we could not collect smoking history data, stage, ECOG performance status, brain metastases, lung metastases, bone metastases, pleural metastasis, adrenal metastasis situation, albumin and MKI67 from 7 patients, the CEA data from 11 patients, LDH from 9 patients, NSE from 20 patients. 

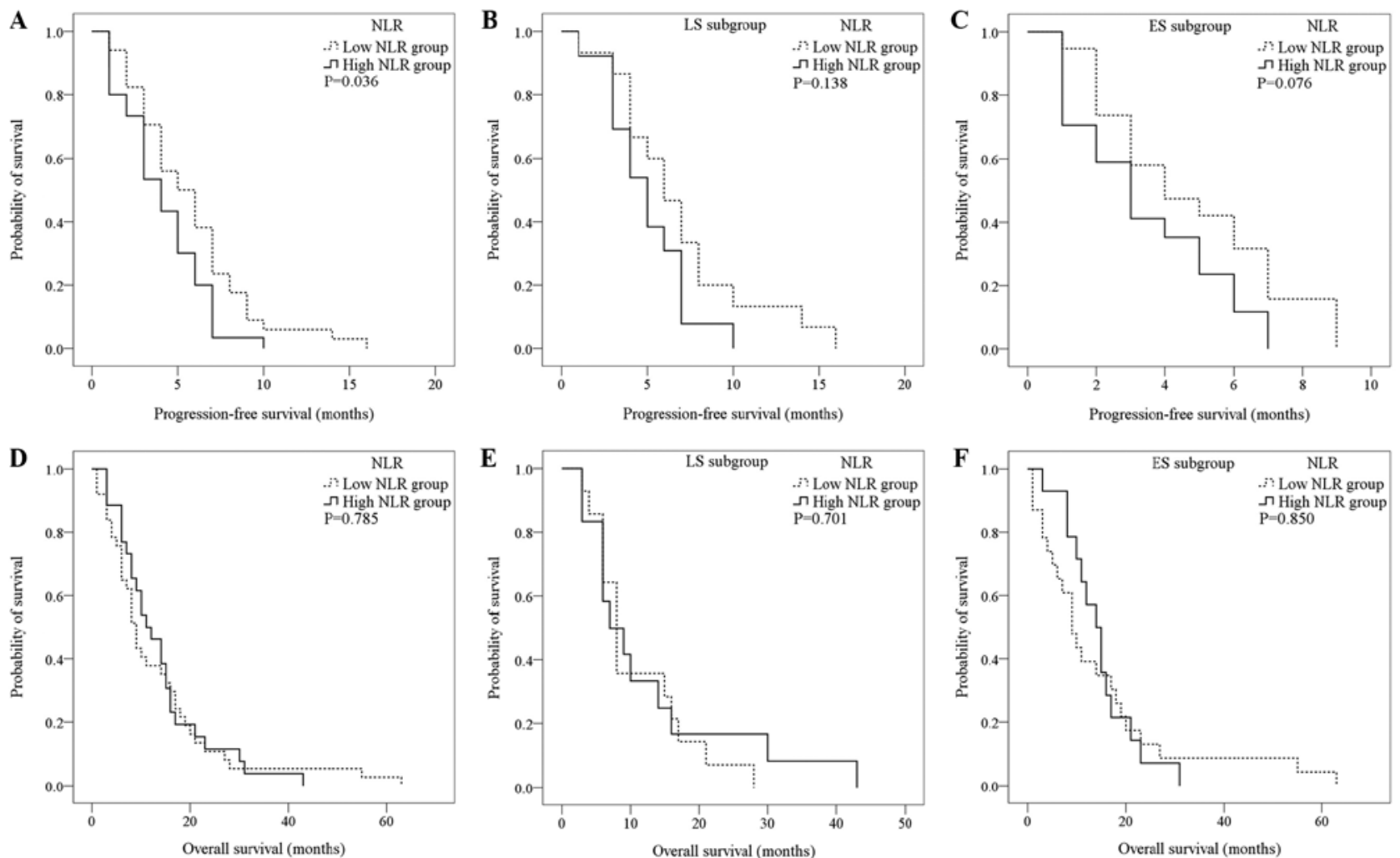

Figure 1. Kaplan-Meier survival curves for PFS and OS for high- and low-NLR patients with small cell lung cancer. (A) Compared with the high-NLR group, the low-NLR group had a longer PFS ( $\mathrm{P}=0.036$; log-rank). (B) In the LS subgroup, the PFS of the low-NLR group was longer than that of the high-NLR group though not significantly ( $\mathrm{P}=0.138$; log-rank). (C) In the ES subgroup, the PFS of the low-NLR group was longer than that of the high-NLR group ( $\mathrm{P}=0.076$; log-rank). (D) The OS time of the high- and low-NLR groups were not significantly different ( $\mathrm{P}=0.785 ;$ log-rank). (E) No significant differences in the OS time of the low- and high-NLR groups in the LS subgroup were observed ( $\mathrm{P}=0.701$; log-rank). (F) No significant differences in the OS time of the low- and high-NLR groups in the ES subgroup were observed ( $\mathrm{P}=0.850$; log-rank). PFS, progression-free survival; OS, overall survival; NLR, neutrophil to lymphocyte ratio; LS, limited stage; ES, extensive stage.

metastasis situation, albumin and MKI67 from 7 patients, the CEA data from 11 patients, LDH from 9 patients, NSE from 20 patients).

Association between PFS, OS time and pre-treatment NLR. Compared with the high-NLR group, the low-NLR group had a longer PFS (5.71 \pm 0.59 vs. $4.10 \pm 0.44$ months; $\mathrm{P}=0.036$; Fig. 1A). In the sub-group analysis of LS and ES patients, the PFS of the low-NLR group was longer than that of the high-NLR group; however, the difference was not statistically significant (Fig. 1B and C). The difference in OS time between the high- $(n=26)$ and low-NLR groups $(n=34)$ was not significantly different $(13.73 \pm 1.87$ vs. $13.22 \pm 2.18$ months; $\mathrm{P}=0.785$; Fig. 1D). A total of 13 patients did not reach the OS time study endpoint. There was no significant difference in the OS time between the high- and low-NLR groups in the ES or LS populations (Fig. 1E and F).

Association between the NLR and the efficacy of the EC or EP regimens. Patients treated with the EP regimen $(n=40)$ had a PFS of $5.36 \pm 0.53$ months. Patients treated with the EC regimen $(n=18)$ had a PFS of $4.78 \pm 0.79$ months. A total of 15 patients were treated with regimens other than EP and EC, including etoposide/lobaplatin or gemcitabine/oxaliplatin, had a PFS of $4.60 \pm 0.68$ months. The difference in survival time was not statistically significant $(\mathrm{P}=0.515)$ (data not shown), when comparing patients treated with EP, patients treated with EC and patients treated with the other regimens.

In the low-NLR group, the PFS of the EP regimen-treated patients $(n=20)$ and the EC regimen-treated patients $(n=10)$ was similar and not significantly different $(5.85 \pm 0.79$ vs. $6.50 \pm 1.09$ months; $\mathrm{P}=0.828$; Fig. 2A). However, in the high-NLR group,the PFS of the EPregimen-treated group $(n=20)$ was significantly longer compared with the $\mathrm{EC}$ regimen-treated group $(n=8 ; 4.59 \pm 0.62$ vs. $2.63 \pm 0.53$ months; $\mathrm{P}=0.021$; Fig. $2 \mathrm{~B})$. This significant difference was also observed in the high-NLR LS group (EP treatment, $6.50 \pm 0.85$ months vs. EC treatment, $2.80 \pm 0.49$ months; $\mathrm{P}=0.002$; Fig. $2 \mathrm{C}$ ), while in the ES group there was no statistically significant difference between the EP and EC treatment groups (EP treatment, 3.54 \pm 0.67 months vs. EC treatment, 2.33 \pm 1.33 months; $\mathrm{P}=0.370$; Fig. 2D).

Among the patients in the EP regimen treated group $(n=40)$, the PFS of the high-NLR group $(n=20)$ and low-NLR group $(n=20)$ was not statistically different $(4.59 \pm 0.62$ vs. $5.85 \pm 0.79$ months; $\mathrm{P}=0.232$; Fig. 2E). However, among the patients in the $\mathrm{EC}$ regimen treated group $(n=18)$, the PFS of the low-NLR group $(\mathrm{n}=10)$ was significantly longer than in the high-NLR group $(\mathrm{n}=8 ; 6.50 \pm 1.09$ vs. $2.63 \pm 0.53 ; \mathrm{P}=0.003$; Fig. $2 \mathrm{~F})$.

Among the total cases that reached the OS time study endpoint, 36 cases had been treated with the EP regimen and 15 cases with the EC regimen with the other 9 patients using neither regimen. There was no statistically 


\section{A}
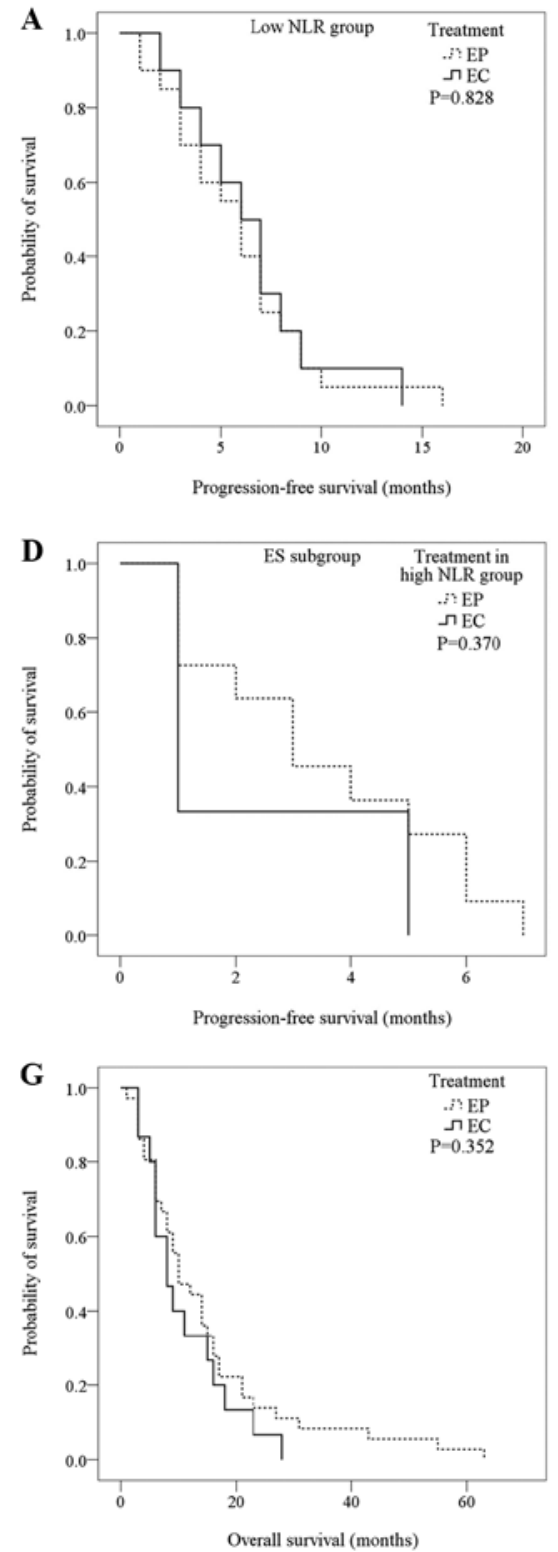
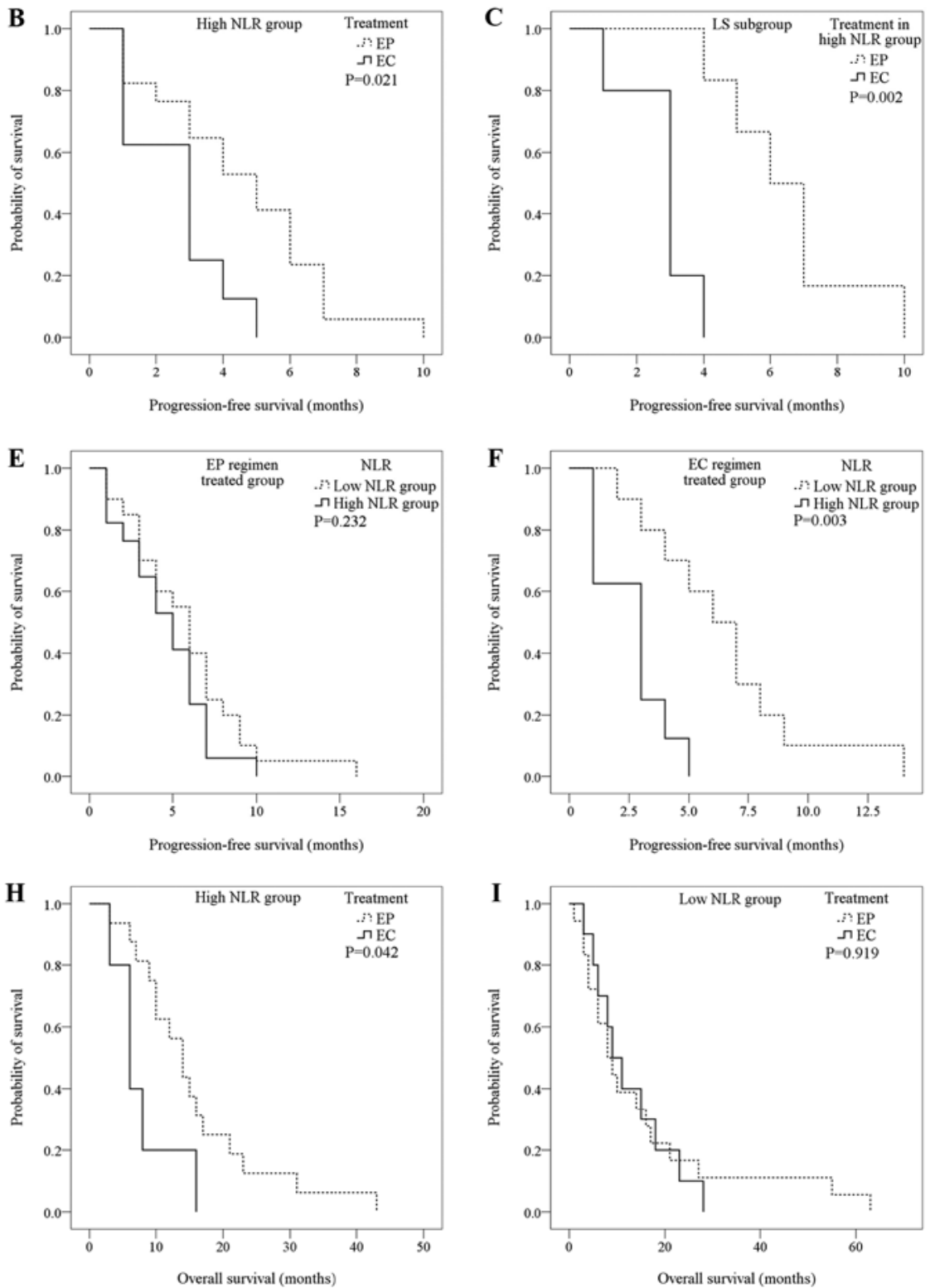

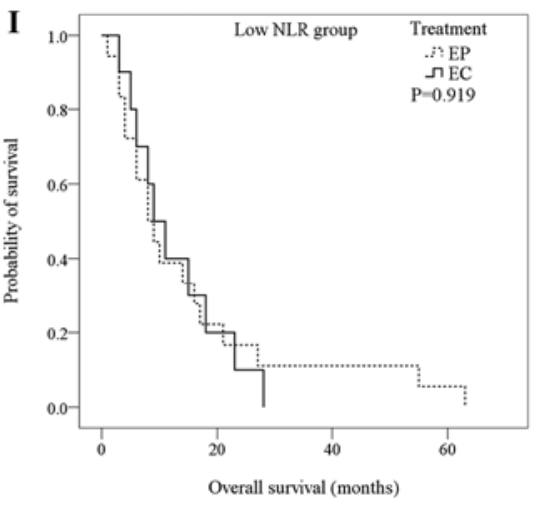

Figure 2. Kaplan-Meier survival curves for the PFS and OS time of patients with small cell lung cancer treated with EP and EC. (A) In the low-NLR group, the PFS of the EP and EC regimen-treated patients was not significantly different ( $\mathrm{P}=0.828$; log-rank). (B) In the high-NLR group, the PFS in the EP treated group was significantly longer compared with the EC treated group ( $\mathrm{P}=0.021$; log-rank). (C) In the LS subgroup, the PFS of the high NLR patients treated with EP was significantly longer than those treated with EC (P=0.002; log-rank). (D) The PFS of the high-NLR patients with treated with EP and EC regimens in the ES subgroup was not significantly different $(\mathrm{P}=0.370$; log-rank). (E) In the EP regimen-treated group, the PFS of the high-group and low-NLR groups was not statistically different ( $\mathrm{P}=0.232$; log-rank). (F) In patients treated with the EC regimen, the low-NLR group had a significantly longer PFS compared with the high-NLR group ( $\mathrm{P}=0.003$; log-rank). $(\mathrm{G})$ The OS time of the EP and EC treated groups was not significantly different ( $\mathrm{P}=0.352$; log-rank). (H) In the high-NLR group, the OS time of the EP regimen-treated patients was significantly longer than that of the EC regimen-treated patients $(\mathrm{P}=0.042$; log-rank). (I) In the low-NLR group, the OS time of the EP and EC regimen-treated patients was similar ( $\mathrm{P}=0.919$; log-rank). PFS, progression-free survival; OS, overall survival; EP, etoposide/cisplatin; EC, etoposide/carboplatin; NLR, neutrophil to lymphocyte ratio; LS, limited stage; ES, extensive stage.

significant difference in OS time (EP, 14.86 \pm 2.34 months vs. EC, $11.00 \pm 1.93$ months; $\mathrm{P}=0.352$; Fig. $2 \mathrm{G}$ ). In the high-NLR group, the OS time of the EP regimen-treated patients $(n=18)$ was significantly longer than that of the EC regimen-treated patients $(n=5) \mathrm{EP}, 15.69 \pm 2.52$ months vs. EC, $7.8 \pm 2.20$ months; $\mathrm{P}=0.042$; Fig. $2 \mathrm{H}$ ). However, in the low-NLR group, the OS time of the EP regimen patients $(n=18)$ and the EC regimen $(n=10)$ was similar and no statistically significant difference was observed (15.28 \pm 4.10 vs. $12.60 \pm 2.60$ months; $\mathrm{P}=0.919$; Fig. 2I). No significant differences in OS time were identified within the low-NLR patients classified as either LS or
ES when comparing treatments with an EP or an EC regimen (LS subgroup, $\mathrm{P}=0.378$; $\mathrm{ES}$ subgroup, $\mathrm{P}=0.052$ ).

The association between NLR and thoracic radiotherapy benefit. A total of 34 patients received local radiotherapy. In the low-NLR group, the PFS of patients who received thoracic radiotherapy $(n=10)$ was significantly longer than for those who did not ( $\mathrm{n}=24 ; 8.20 \pm 1.35$ vs. $4.67 \pm 0.50$ months; $\mathrm{P}=0.011$; Fig. 3A). The increase in PFS was greater in the low-NLR LS

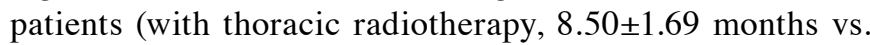
without thoracic radiotherapy, 5.00 \pm 0.69 months; $\mathrm{P}=0.049$; 

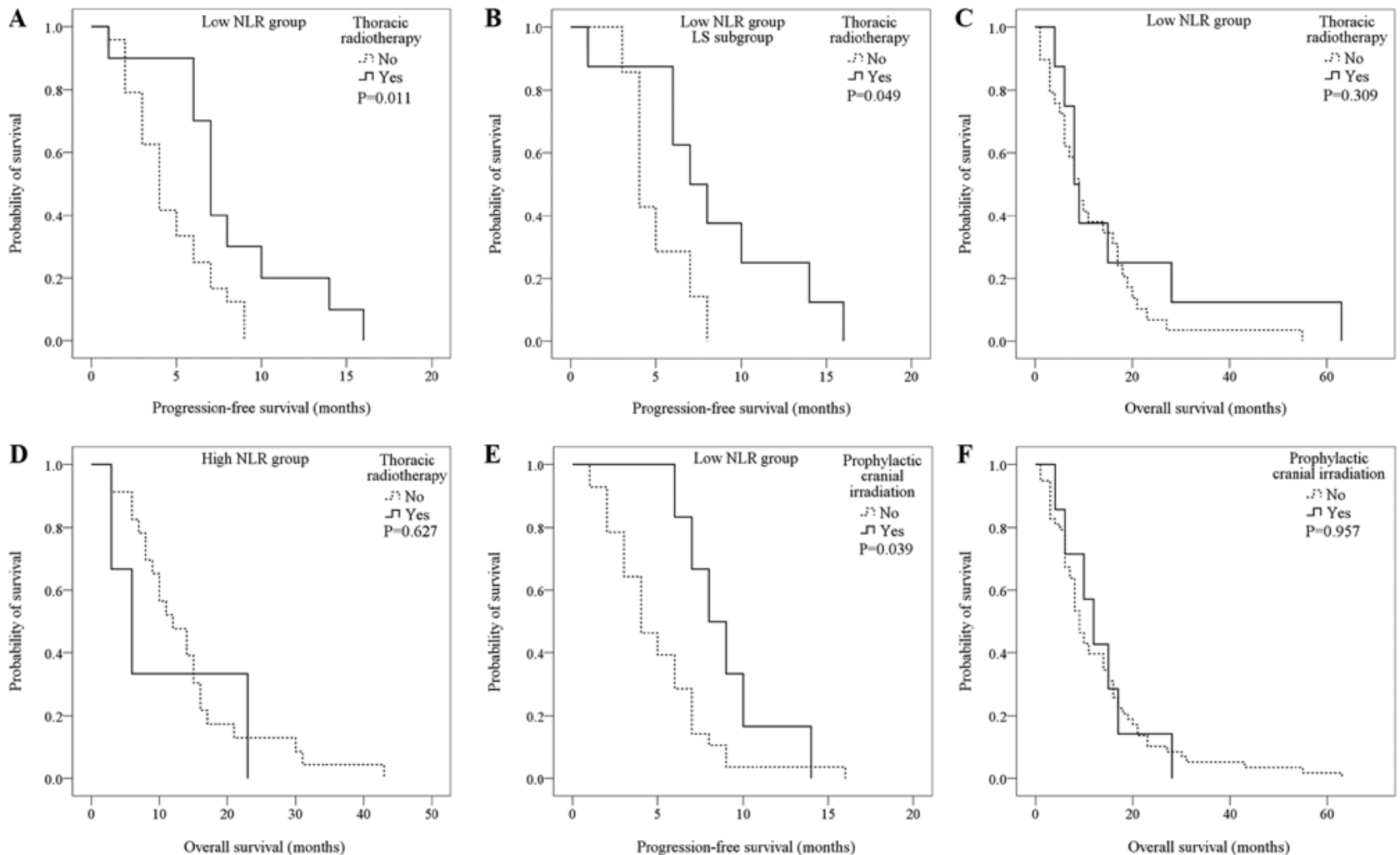

Figure 3. Kaplan-Meier survival curves for the PFS and OS time of patients with small cell lung cancer patients who received radiotherapy and those that did not. (A) Patients in the low-NLR group that received thoracic radiotherapy had a longer PFS compared with those who did not (P=0.011; log-rank). (B) LS patients in the low-NLR group who received radiotherapy had a longer PFS compared with LS patients in the low-NLR group who did not receive radiotherapy ( $\mathrm{P}=0.049$; log-rank). (C) Among the patients in the low-NLR group who reached the OS time study endpoint, those who received thoracic radiotherapy had a longer OS than those who did not ( $\mathrm{P}=0.309$; log-rank). (D) In the high-NLR group, the OS time was not statistically different between patients who received thoracic radiotherapy and those that $\operatorname{did} \operatorname{not}(\mathrm{P}=0.627$; log-rank). (E) In the low-NLR group, patients who received prophylactic cranial irradiation had a longer PFS than those who did not $(\mathrm{P}=0.039$, log-rank). (F) The OS time of patients who received prophylactic cranial irradiation or not $(\mathrm{P}=0.957$; $\log$-rank) in patients who received prophylactic cranial irradiation vs. patients who did not receive prophylactic cranial irradiation. PFS, progression-free survival; OS, overall survival; EP, etoposide/cisplatin; EC, etoposide/carboplatin; NLR, neutrophil to lymphocyte ratio; LS, limited stage; ES, extensive stage.

Fig. 3B), while with the ES patients, no statistically significant difference was determined $(7.00 \pm 0.00$ vs. $4.53 \pm 0.65$ months; $\mathrm{P}=0.439$ ) (data not shown). In the high-NLR group, there were no significant differences between patients who received radiotherapy and those who did not $(6.67 \pm 0.33$ vs. $3.82 \pm 0.45$ months; $\mathrm{P}=0.095$ ) (data not shown).

Among the patients in the low-NLR group who reached the OS time study endpoint, those who received thoracic radiotherapy $(n=8)$ had a longer OS time than those who did not $(n=26)$, although the difference was not statistically significant $(17.63 \pm 7.01$ vs. $12.00 \pm 2.05$ months; $\mathrm{P}=0.309$; Fig. $3 \mathrm{C})$. Receiving or not receiving thoracic radiotherapy did not result in a statistically significant difference in the OS time in the LS $(11.50 \pm 3.63$ vs. $10.62 \pm 2.28$ months; $\mathrm{P}=0.804)$ or $\mathrm{ES}$ sub-groups (9.25 \pm 2.71 vs. $8.02 \pm 3.75$ months; $\mathrm{P}=0.136$ ) (data not shown). In the high-NLR group, patients who received radiotherapy had a similar OS time to those that did not receive radiotherapy (10.67 \pm 6.22 vs. $14.13 \pm 1.99$ months; $P=0.627$; Fig. $3 D$ ).

Association between NLR, cranial radiotherapy and PFS. Analysis of 34 patient cases without cranial metastasis who received prophylactic cranial irradiation, a SCLC treatment $(4,19)$, following chemotherapy demonstrated that low-NLR patients who received prophylactic cranial irradiation $(n=6)$ had a longer PFS than those who did not $(n=28 ; 9.00 \pm 1.16$ vs. 5.00 \pm 0.60 months; $\mathrm{P}=0.039$; Fig. $3 \mathrm{E}$ ). In the high-NLR group, there were no significant differences between patients who received prophylactic cranial irradiation $(\mathrm{n}=2)$ and did not $(n=28)(6.00 \pm 1.00$ vs. $3.96 \pm 0.45$ months; $P=0.353)$. There was no statistically significant difference observed in the OS time between patients who received prophylactic cranial irradiation and those who did not $(13.14 \pm 3.03$ vs. $13.16 \pm 1.59$ months; $\mathrm{P}=0.957$; Fig. 3F).

\section{Discussion}

The identification of cancer driver genes and the emergence of targeted drug resulted in remarkable progress in recent years in the field of non-small cell lung cancer treatment. However, little has been achieved in the treatment of SCLC, with chemotherapy still being the main therapy approach (20). SCLC is sensitive to chemotherapy and radiotherapy, and previous studies have demonstrated that chemotherapy relieves symptoms and improves survival time for the majority of patients with SCLC $(21,22)$. To date, the regimen, which consists of etoposide in combination with platinum, remains the first recommended regimen to treat SCLC (23).

Carboplatin has a similar therapeutic effect to cisplatin, but has fewer side effects and is better tolerated (24-26). In multiple randomized controlled clinical studies (24-26), carboplatin or 
cisplatin in combination with etoposide had a similar clinical outcome. A meta-analysis of 663 cases compared the curative effects of carboplatin and cisplatin in SCLC, and determined that for either LS or ES patients, the PFS (5.5 vs. 5.3 months) and the OS time (9.6 vs. 9.4 months) of the two regimens were not significantly different (7). In the present study, the PFS was compared in patients receiving EP, EC and other regimens. It was determined that the difference in PFS was not significant $(\mathrm{P}=0.44)$ (data not shown). Deciding which treatment option is optimal remains challenging.

A higher NLR level may be associated with an increase in neutrophils and/or a decrease in lymphocytes. Neutrophils regulate the activity of lymphocytes or natural killer cells, thus inhibiting tumor growth (27). By contrast, lymphocytes may induce the death of cytotoxic cells, and suppress the anti-tumor immune response $(28,29)$. In addition, a previous study reported that wide deposition of abscess-induced neutrophil extracellular traps (NETs) may segregate circulating tumor cells, promoting the development of tumor metastasis (30). NETs may also protect circulating tumor cells from being purged by the immune system by adhering to and recruiting platelets (31). The aforementioned studies serve as the theoretical basis for the evaluation of tumor prognosis using NLR. Previous studies investigating immunocytes in the tumor microenvironment have demonstrated that tumor-associated macrophages (TAMs) and tumor-associated neutrophils (TANs) are involved in tumor genesis and development (32-34). TAMs are divided into M1- and M2-types, while TANs are divided into N1- and N2-types, with M2- and N2-types serving important roles in promoting tumor genesis, development and metastasis (32-34). Although current hematological analysis do not distinguish tumor-associated neutrophil subtypes, an increase in the neutrophil ratio and, therefore, an increase in the NLR has been reported when examining blood samples from patients with a TAN ratio increase. Thus, the NLR increase demonstrated in the current study may be a TAN ratio increase. It would follow that the prognosis of patients with SCLC may be affected by the increase of TANs, which may affect tumor proliferation and drug resistance, manifesting as an NLR in hematological analysis.

Deng et al (35) reported that a high NLR results in a shorter PFS when investigating SCLC. Xie et al (36) and Hong et al (37) demonstrated that a high NLR may predict poor prognosis for patients with SCLC. These results are similar to the results obtained in the present study, in which patients with a NLR of $\leq 3.80$ (low NLR) achieved a significantly better curative effect and longer PFS and OS when treated with the EP regimen, compared with the EC regimen, with an even better PFS observed in the LS patients. The PFS of the low-NLR patients was longer in LS patients receiving the $\mathrm{EC}$ regimen. These results suggested that EP and EC regimens had an equal effect in the low-NLR group patients, whereas in the high-NLR group the EP regimen was superior to the EC regimen; which was likely affected by the tumor microenvironment. The tumor microenvironment contains cytokines produced by TAMs and TANs that may influence carboplatin. Wang et al (38) revealed that interleukin 6 (IL6) decreases carboplatin treatment sensitivity through activating the signal transducer and activator of transcription 3 (STAT3) signaling pathway. Thus, it was hypothesized that patients with a higher
NLR had a higher ratio of TAMs and TANs in the present study. This may resulted in the presence of more cytokines, including IL6, to activate drug resistance signaling pathways against carboplatin, including the STAT3 signaling pathway, in order to induce drug resistance. This warrants further investigation and monitoring the NLR may aid the selection of a first line chemotherapy regimen.

Radiotherapy is another key method used to treat patients with SCLC. Currently, thoracic radiotherapy is the standard treatment for patients with LS SCLC (39). The present study demonstrated that the low-NLR group patients had a longer PFS following thoracic radiotherapy. Both thoracic and cranial radiotherapy are local treatments, so higher NLR levels may be associated with systemic tumor metastasis, tumor progression and tumor drug resistance. Thus, it is possible that local treatment is more effective at improving the prognosis of patients in the low-NLR group.

In conclusion, for the initially diagnosed patients with SCLC, pre-treatment NLR may have prognostic prediction value. In addition, it may aid in optimal first-line therapy selection. The current study revealed that compared with the high-NLR group, the low-NLR group had a longer PFS. Patients with high pre-treatment NLR may benefit from EP over EC treatment. A limitation of the present study was its retrospective nature and the relatively small number of patients due to morbidity and patients lost to follow-up. Future prospective clinical studies with a larger sample size focusing on the underlying cellular mechanisms are required to determine the significance of NLR in the treatment of SCLC.

\section{Acknowledgements}

The authors would like to thank Professor Qiang Chen (Departments of Oncology, Fujian Medical University Union Hospital) for his contribution to the research.

\section{Funding}

The present study was supported by funding from the Fujian Province Natural Genetic Health Joint Capital Project awarded to Chunmei Shi (grant no. 2015J01397) and the Young and Middle-Aged Teachers' Scientific Research Project in Fujian Province awarded to Zhangchi Pan (grant no. JAT170226).

\section{Availability of data and materials}

The datasets used and/or analyzed during the current study are available from the corresponding author on reasonable request.

\section{Authors' contributions}

ZCP and LZ contributed to the conception of the study, analyzed the data and wrote the manuscript. CL, XBH, SFS, XYL and CMS contributed to the design of the study, and conducted additional analyses. All authors read and approved the final manuscript.

\section{Ethics approval and consent to participate}

All procedures performed in the current study were approved by the Institutional Review Board of Fujian Medical University 
Union Hospital (Institutional Review Board no. 2017KY084). Informed consent was obtained from patients included in the study.

\section{Patient consent for publication}

Not applicable.

\section{Competing interests}

The authors declare that they have no competing interests.

\section{References}

1. Torre LA, Bray F, Siegel RL, Ferlay J, Lortet-Tieulent J and Jemal A: Global cancer statistics, 2012. CA Cancer J Clin 65: 87-108, 2015

2. Klutker G, Sauer R and Fietkau R: Combined treatment modality in small cell lung cancer: The impact of radiotherapy on survival. Strahlenther Onkol 184: 61-66, 2008.

3. Kurup A and Hanna NH: Treatment of small cell lung cancer. Crit Rev Oncol Hematol 52: 117-126, 2004.

4. Puglisi M, Dolly S, Faria A, Myerson JS, Popat S and O'Brien ME: Treatment options for small cell lung cancer-do we have more choices? Br J Cancer 102: 629-638, 2010.

5. Gaspar LE, Mcnamara EJ, Gay EG, Putnam JB, Crawford J, Herbst RS and Bonner JA: Small-cell lung cancer: Prognostic factors and changing treatment over 15 years. Clin Lung Cancer 10: 115-122, 2009.

6. Murray N and Turrisi AT III: A review of first-line treatment for small-cell lung cancer. J Thorac Oncol 1: 270-278, 2006.

7. Rossi A, Di Maio M, Chiodini P, Rudd RM, Okamoto H, Skarlos DV, Früh M, Qian W, Tamura T, Samantas E, et al: Carboplatin- or cisplatin-based chemotherapy in first-line treatment of small-cell lung cancer: The COCIS meta-analysis of individual patient data. J Clin Oncol 30: 1692-1698, 2012.

8. O'Dwyer PJ and Calvert AH: Platinum analogs. In: Cancer: Principles and Practice of Oncology. DeVita VT, Lawrence TS and Rosenbery SA (eds). 10th edition. Lippincott Williams \& Wilkins, Philidelphia, PA, pp199-207, 2015.

9. Go RS and Adjei AA: Review of the comparative pharmacology and clinical activity of cisplatin and carboplatin. J Clin Oncol 17: 409-422, 1999.

10. O'Callaghan DS, O'Donnell D, O'Connell $\mathrm{F}$ and O'Byrne KJ: The role of inflammation in the pathogenesis of non-small cell lung cancer. J Thorac Oncol 5: 2024-2036, 2010.

11. Gondo T, Nakashima J, Ohno Y, Choichiro O, Horiguchi Y, Namiki K, Yoshioka K, Ohori M, Hatano T and Tachibana M: Prognostic value of neutrophil-to-lymphocyte ratio and establishment of novel preoperative risk stratification model in bladder cancer patients treated with radical cystectomy. Urology 79: 1085-1091, 2012.

12. Mano Y, Shirabe K, Yamashita Y, Harimoto N, Tsujita E, Takeishi K, Aishima S, Ikegami T, Yoshizumi T, Yamanaka T and Maehara Y: Preoperative neutrophil-to-lymphocyte ratio is a predictor of survival after hepatectomy for hepatocellular carcinoma: A retrospective analysis. Ann Surg 258: 301-305, 2013.

13. Zheng YB, Zhao W, Liu B, Lu LG, He X, Huang JW, Li Y and Hu BS: The blood neutrophil-to-lymphocyte ratio predicts survival in patients with advanced hepatocellular carcinoma receiving Sorafenib. Asian Pac J Cancer Prev 14: 5527-5531, 2013.

14. Yao Y, Yuan D, Liu H, Gu X and Song Y: Pretreatment neutrophil to lymphocyte ratio is associated with response to therapy and prognosis of advanced non-small cell lung cancer patients treated with first-line platinum-based chemotherapy. Cancer Immunol Immunother 62: 471-479, 2013

15. Unal D, Eroglu C, Kurtul N, Oguz A and Tasdemir A: Are neutrophil/lymphocyte and platelet/lymphocyte rates in patients with non-small cell lung cancer associated with treatment response and prognosis? Asian Pac J Cancer Prev 14: 5237-5242, 2013.

16. Kacan T, Babacan NA, Seker M, Yucel B, Bahceci A, Eren AA, Eren MF and Kilickap S: Could the neutrophil to lymphocyte ratio be a poor prognostic factor for non small cell lung cancers? Asian Pac J Cancer Prev 15: 2089-2094, 2014.
17. Eisenhauer EA Therasse P, Bogaerts J, Schwartz LH, Sargent D, Ford R, Dancey J, Arbuck S, Gwyther S, Mooney M, et al: New response evaluation criteria in solid tumours: Revised RECIST guideline (version 1.1). Eur J Cancer 45: 228-247, 2009.

18. Micke P, Faldum A, Metz T, Beeh KM, Bittinger F, Hengstler JG and Buhl R: Staging small cell lung cancer: Veterans administration lung study group versus international association for the study of lung cancer-what limits limited disease? Lung Cancer 37: 271-276, 2002.

19. Naidoo J, Kehoe M, Sasiadek W, Hacking D and Calvert P: Prophylactic cranial irradiation in small cell lung cancer: A single institution experience. Ir J Med Sci 183: 129-132, 2014

20. Byers LA and Rudin CM: Small cell lung cancer: Where do we go from here? Cancer 121: 664-672, 2015.

21. Ettinger DS and Aisner J: Changing face of small-cell lung cancer: Real and artifact. J Clin Oncol 24: 4526-4527, 2006.

22. van Meerbeeck JP, Fennell DA and De Ruysscher DK: Small-cell lung cancer. Lancet 378: 1741-1755, 2011.

23. Evans W, Shepherd FA, Feld R, Osoba D, Dang P and Deboer G: VP-16 and cisplatin as first-line therapy for small-cell lung cancer. J Clin Oncol 3: 1471-1477, 1885.

24. Hatfield LA, Huskamp HA and Lamont EB: Survival and toxicity after cisplatin plus etoposide versus carboplatin plus etoposide for extensive-stage small-cell lung cancer in elderly patients. J Oncol Pract 12: 666-673, 2016.

25. Skarlos DV, Samantas E, Kosmidis P, Fountzilas G, Angelidou M, Palamidas P, Mylonakis N, Provata A, Papadakis E, Klouvas G, et al: Randomized comparison of etoposide-cisplatin vs. etoposide-carboplatin and irradiation in small-cell lung cancer. A Hellenic Co-operative Oncology Group study. Ann Oncol 5: 601-607, 1994.

26. Okamoto H, Watanabe K, Kunikane H, Yokoyama A, Kudoh S, Asakawa T, Shibata T, Kunitoh H, Tamura T and Saijo N: Randomised phase III trial of carboplatin plus etoposide vs split doses of cisplatin plus etoposide in elderly or poor-risk patients with extensive disease small-cell lung cancer: JCOG 9702. Br J Cancer 97: 162-169, 2007.

27. Pillar J, Kamp VM, van Hoffen E, Visser T, Tak T, Lammers JW, Ulfman LH, Leenen LP, Pickkers P and Koenderman L: A subset of neutronphils in human systemic inflammation inhibits t cell responses through mac-1. J Clin Invest 122: 327-336, 2012.

28. Coussens LM and Werb Z: Inflammation and cancer. Nature 420 : 860-867, 2002.

29. Mantovani A, Allavena P, Sica A and Balkwill F: Cancer-related inflammation. Nature 454: 436-444, 2008.

30. Cools-Lartigue J, Spicer J, McDonald B, Gowing S, Chow S, Giannias B, Bourdeau F, Kubes P and Ferri L: Neutrophil extracellular traps sequester circulating tumor cells and promote metastasis. J Clin Invest 123: 3446-3458, 2013.

31. Demers M and Wagner DD: Neutrophil extracellular traps: A new link to cancer-associated thrombosis and potential implications for tumor progression. Oncoimmunology 2: 22946, 2013.

32. Sica A and Mantovani A: Macrophage plasticity and polarization: In vivo veritas. J Clin Invest 122: 787-795, 2012.

33. Biswas SK and Mantovani A: Macrophage plasticity and interaction with lymphocyte subsets: Cancer as a paradigm. Nat Immunology 11: 889-896, 2010.

34. Allavena P, Sica A, Garlanda C and Mantovani A: The Yin-Yang of tumor-associated macrophages in neoplastic progression and immune surveillance. Immunol Rev 222: 155-161, 2008.

35. Deng M, Ma X, Liang X, Zhu C and Wang M: Are pretreatment neutrophil-lymphocyte ratio and platelet-lymphocyte ratio useful in predicting the outcomes of patients with small-cell lung cancer? Oncotarget 8: 37200-37207, 2017.

36. Xie D, Marks R, Zhang M, Jiang G, Jatoi A, Garces Y, Mansfield A, Molina J and Yang P: Nomograms predict overall survival for patients with small-cell lung Cancer incorporating pretreatment peripheral blood markers. J Thorac Oncol 10: 1213-1220, 2015

37. Hong X, Cui B, Wang M, Yang Z, Wang L and Xu Q: Systemic immune-inflammation index, based on platelet counts and neutrophil-lymphocyte ratio, is useful for predicting prognosis in small cell lung cancer. Tohoku J Exp Med 236: 297-304, 2015.

38. Wang ZY, Zhang JA, Wu XJ, Liang YF, Lu YB, Gao YC, Dai YC, Yu SY, Jia Y, Fu XX, et al: IL-6 inhibition reduces STAT3 activation and enhances the antitumor effect of carboplatin. Mediators Inflamm 2016: 8026494, 2016.

39. Pignon JP, Arriagada R, Ihde DC, Johnson DH, Perry MC, Souhami RL, Brodin O, Joss RA, Kies MS, Lebeau B, et al: A meta-analysis of thoracic radiotherapy for small-cell lung cancer. New Engl J Med 327: 1618-1624, 1992. 